Check for updates

The BMJ

Cite this as: BMJ2021;373:n1659 http://dx.doi.org/10.1136/bmj.n1659 Published: 29 June 2021

\title{
Covid-19: Third vaccine dose boosts immune response but may not be needed, say researchers
}

\section{Elisabeth Mahase}

The interval between the first and second doses of the Oxford AstraZeneca vaccine can be extended up to 10 months and a third dose of the vaccine provides a strong boost to the immune response, according to preliminary results.

In a preprint, published on 28 June, ${ }^{1}$ researchers from the University of Oxford reported that extending the interval between the first and second dose to 45 weeks resulted in higher antibody titres. They also found that a third dose given 44 to 45 weeks after the second increased antibody titres further, and that adverse events were lower after the second or third dose than after the first.

Researchers said the results are positive, especially for areas affected by vaccine shortages, as they could provide "greater flexibility in vaccination schedules." But they said that currently there is no indication that a booster is needed and stressed that the "urgent priority" is to ensure people around the world receive their first dose.

The study included volunteers aged 18-55 years from earlier trials of the vaccine-who had received either a single dose or two doses-who were then invited back for vaccination in March 2021.

\section{Delayed second dose}

The researchers found that longer dose intervals between the first and second dose led to higher antibody levels. They reported that for those who had eight to 12 weeks between dose one and two (median age 39), the median antibody level 28 days after the second dose was 923 tIgG EU. For those who had a 15 to 25 week interval (median age 36), it was 1860 tIgG EU and for the 44 to 45 week interval (median age 32) it was 3738 tIgG EU.

Six months after the second dose, antibody levels remained significantly higher in the group who had a 15 to 25 week interval between doses compared with the eight to 12 week interval group-median 1240 tgG EU and 278 tIgG EU respectively. The data are not yet available for the 44 to 45 week interval.

The team added that the IgG binding titres to the four variants tested (D614G, alpha, beta, and delta) were "significantly greater" after the second dose than before the second dose.

\section{Booster dose}

For the booster (third) dose, 75 participants who had their first two doses with an interval of eight to 16 weeks were assessed. The preprint reported that antibody levels 28 days after the third dose were significantly higher than 28 days after the second dose-3746 tIgG EU and 1792 respectively.
The team also found that binding antibody titres to the beta variant "increased significantly" after the third dose, while neutralising antibody titres following the booster were higher than those after the second dose against alpha, beta, and delta variants.

In the UK, the government has said it plans to roll out a booster vaccine at the start of this autumn, in order to protect the most vulnerable ahead of winter. ${ }^{2}$

But speaking at a Science Media Centre briefing on the preprint, Andrew Pollard, the clinical trials lead for the vaccine and director of the Oxford Vaccine Group, said, “There's no indication today that we need boosters. It is something where we need to keep looking at the data and make decisions as the months go by.

Pollard added, "We have countries facing huge waves of disease at this moment with a largely unvaccinated population. We saw the new very sharp increase of cases in Bangladesh over the weekend. And that is a situation where getting the first dose to more people as soon as possible is the most urgent priority. Before third doses are given, we should be trying to make sure all those vulnerable people-the older adults and those with other health conditions, all around the world-are protected. That's the most urgent thing to do."

1 Flaxman A, Marchevsky N, Jenkin D, et al. Tolerability and immunogenicity after a late second dose or a third dose of ChAdOX1 nCoV-19 (AZD1222).SSRN [Preprint]. 2021https://papers.ssrn.com/sol3/papers.cfm?abstract_id=3873839doi: 10.2139/ssrn.3873839.

2 Mahase E. Covid-19: Booster vaccine to be rolled out in autumn as UK secures 60m more Pfizer doses. BM/2021;373:n1116. doi: 10.1136/bmj.n1116 pmid: 33926905

This article is made freely available for use in accordance with BMJ's website terms and conditions for the duration of the covid-19 pandemic or until otherwise determined by BMJ. You may use, download and print the article for any lawful, non-commercial purpose (including text and data mining) provided that all copyright notices and trade marks are retained. 